\title{
Can Controlled Vestibular Stimulation Delay Brain Aging?
}

\author{
Kumar Sai Sailesh ${ }^{1}$,Rose Usha ${ }^{2}$, Padmanabha $^{3}{ }^{3}$ Jobby Abraham ${ }^{4}$,Mukkadan J K ${ }^{* 5}$ \\ ${ }^{1}$ Research scholar, Little Flower Medical research Centre, Angamaly, Kerala, India \\ ${ }^{2}$ Assoc professor, Dept of Anatomy, LIMSAR, Angamaly. \\ ${ }^{3}$ Asst professor, Dept of Physiology, Jazan University, Saudi Arabia \\ ${ }^{4}$ Vice Principal, Travancore Medical College, Kollam. \\ ${ }^{5}$ Research director, Little Flower Medical Research Centre, Angamaly, Kerala, India
}

\section{Abstract}

Aging is believed to be a first-order risk factor for most neurodegenerative disorders. Brain changes do not occur to the same extent in all brain regions.7 Men and women may also differ with frontal and temporal lobes most affected in men compared with the hippocampus and parietal lobes in women. The neurotransmitters most often discussed with regard to ageing are dopamine, serotonin and acetyl-choline. Vestibular stimulation modulates the neuro-transmitters which are involved in brain aging and delay aging. Hence we recommend controlled vestibular stimulation to all. This in the need of time to identify the importance of vestibular system and to start translational research in this area.

Key Words: Brain aging; Controlled Vestibular Stimulation; Neuro-transmitters

INTRODUCTION

timulating vestibular system by controlling direction, $\checkmark$ duration, frequency and intensity. Ideal direction, duration, frequency and intensity are yet to be determined. Hammam E etal suggested that low frequency stimulation is benefitial.[1] Winter etal in reported that they have observed decrease in salivary cortisol in volunteers who subjected to front to back motion on a hexapod.[2] As people age, they change in a myriad of ways - both biological and psychological. Some of these changes may be for the better, and others are not. Aging causes changes to the brain size, vasculature, and cognition. The brain shrinks with increasing age and there are changes at all levels from molecules to morphology. Incidence of stroke, white matter lesions, and dementia also rise with age, as does level of memory impairment and there are changes in levels of neurotransmitters and hormones.[6] Aging is believed to be a first-order risk factor for most neurodegenerative disorders. Brain changes do not occur to the same extent in all brain regions.[7] Men and women may also differ with frontal and temporal lobes most affected in men compared with the hippocampus and parietal lobes in women. $[8,9]$ The most widely seen cognitive change associated with ageing is that of memory. Memory function can be broadly divided into four sections, episodic memory, semantic memory, procedural memory, and working memory.[10]

The neurotransmitters most often discussed with regard to ageing are dopamine and serotonin. Dopamine levels decline by around $10 \%$ per decade from early adulthood and have been associated with declines in cognitive and motor performance.[11, 12] Serotonin and brain derived neurotrophic factor levels also fall with increasing age and may be implicated in the regulation of synaptic plasticity and neurogenesis in the adult brain.[16] A substance related to neurotransmitter levels, monoamine oxidase, increases with age and may liberate free radicals from reactions that exceed the inherent antioxidant reserves.[17] Other factors that have been implicated in the aging brain include

\author{
Address for correspondence* \\ Dr. J.k. mukkadan \\ Research Director, \\ Little Flower Medical Research Centre,Angamaly,Kerala, \\ India.drmukkadan@sify.com
}

calcium dysregulation,[18] mitochondrial dysfunction, and the production of reactive oxygen species.[19]

Another factor to consider with regard to the aging brain and its cognitive performance is hormonal influence. It is known that sex hormones can affect cognitive processes in adulthood and that changes in sex hormones occur in ageing particularly in women at menopause. Women also have a higher incidence of Alzhimer's disease (AD) even when longer life expectancy is taken into account.[13] Growth hormone levels also decline with age and may be associated with cognitive performance although the evidence is far from clear.[6]

The neuronal cell loss that occurs with aging has been partly attributed to increased production of nitric oxide and high caspase activity. Exogenous MLT administration might delay brain aging (by moderation of death of neurons and glia) via decreasing the nitrite/nitrate level.[3] Vestibular system is also having projections to Suprachiasmatic Nucleus and raphe nucleus.[4]The circadian release of the hormone melatonin is regulated by the suprachiasmatic nucleus $(\mathrm{SCN})$, which feeds back into the nucleus to modulate sleep and circadian phase through activation of the $\mathrm{MT}(1)$ and/or $\mathrm{MT}(2)$ melatonin receptors.[5]

The purpose of this article is to review research reports related to vestibular stimulation and its role in brain aging, with the intent of clarifying the present knowledge base in this area, and suggesting future research needs.

\section{MATERIALSAND METHODS}

Searches of the review study register articles from google.com, pubmed.com, British medical journal.com, Medline, ERIC, frontiersin.org and online standardized journals.

\section{How controlled vestibular stimulation delays brain ageing?}

Controlled vestibular stimulation delays brain ageing trough serotonin and melatonin

Serotonin is widely distributed throughout the central nervous system and is implicated in a variety of neural functions such as pain, feeding, sleep, sexual behavior, cardiac regulation and cognition.Age-related alterations in serotonin function may increase the vulnerability to psychiatric and neurodegenerative disorders in late life.[14] Positron emission tomography has 
demonstrated specific aging reductions in dopamine and serotonin (5-hydroxytryptamine [5-HT]) receptor subtypes. Depression has been widely attributed to deficient 5-HT neurotransmission. It is also worth noting that there was a $30 \%$ to $40 \%$ reduction in the concentration of the 5-HT metabolite, 5hydroxyindoleacetic acid (5-HIAA), in the ventricular CSF of these depressed patients, underlining the possible relationship between disturbances of serotonergic neurotransmission and depressive symptoms.[20] Vestibular stimulation relieves stress[22] and increases serotonin release[21] and delays brain ageing. In the pinealocyte cells of the pineal gland, serotonin Nacetyltransferase is involved in the conversion of serotonin to melatonin. Serotonin N-acetyltransferase may contribute to multifactorial genetic diseases such as altered behavior in sleep/wake cycle and mood disorders.[23] Vestibular system is also having projections to Suprachiasmatic Nucleus and raphe nucleus. The circadian release of the hormone melatonin is regulated by the suprachiasmatic nucleus $(\mathrm{SCN})$, which feeds back into the nucleus to modulate sleep and circadian phase through activation of the MT (1) and/or MT (2) melatonin receptors.24 Exogenous MLT administration might delay brain aging (by moderation of death of neurons and glia) via decreasing the nitrite/nitrate level.3

Controlled vestibular stimulation delays brain ageing trough dopamine Drugs which are used for treatment of Parkinson's disease like L-DOPA will increase the dopamine levels, which leads to further complications like schizophrenia. In contrast vestibular stimulation treats parkinson's disease Figure Vestibular pathways that may influence basal ganglia transmission. RF: reticular formation, SNc: Substantia nigra parscompacta, SNr: Substantia nigra pars reticulata, STN: subthalamic nucleus, SVS: stochastic vestibular stimulation, Thal: thalamus.

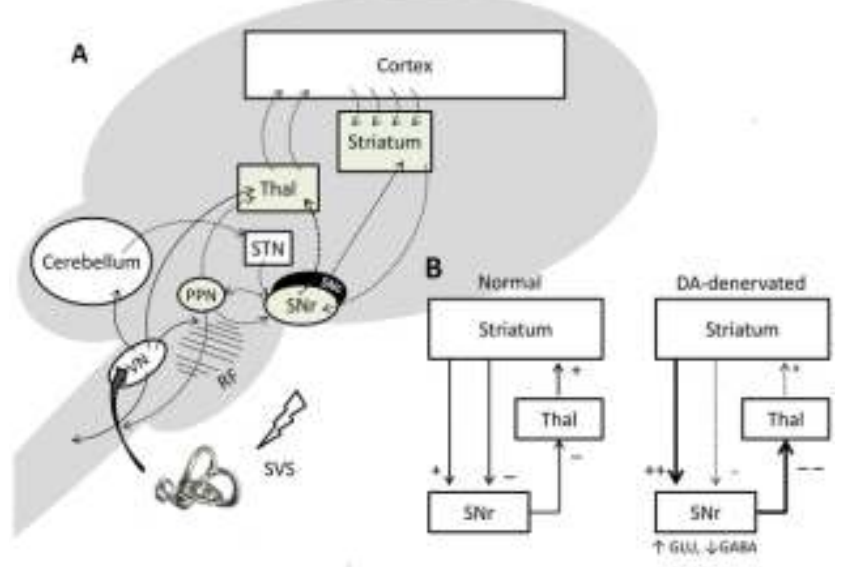

without altering dopamine levels.[25]

Controlled vestibular stimulation delays brain ageing trough acetylcholine

As a result of the findings cited in both humans and animals, the primary therapeutic approach to date to address the cognitive loss associated with $\mathrm{AD}$ has been that of a cholinergic replacement strategy. This approach has been attempted using muscarinic and nicotinic-cholinergic ligands and acetylcholinesterase inhibitors.[26, 27] To date, however, only the data derived from clinical trials with acetylcholinesterase inhibitors (e.g., tacrine, donepezil, rivastigmine, and galantamine) have provided convincing evidence of an adequate level of efficacy and reliability in $\mathrm{AD}$ balanced with an acceptable burden of side effects. Accordingly, these agents are the only drugs currently approved by the United States for clinical use in $\mathrm{AD}$. Due to the modest risk of hepatotoxicity associated with tacrine, the latter three compounds listed above are generally preferred. Agents such as the glutamate antagonist memantine have recently been associated with improvements in advanced $\mathrm{AD}$ symptomatology and may suggest one new approach to therapy. Caloric stimulation with hot water increased the glutamate release in the MVN, while that with ice water decreased it. It is evidenced that glutamate is a neurotransmitter between afferent vestibular nerve and the MVN. Electrical stimulation of the round window evoked the release of hypothalamic histamine and hippocampal $\mathrm{ACh}$ and these effects were inhibited by the blockade of second-order vestibular neurons by the pre-injection of 6,7-dinitroquinoxaline-2,3-dione (DNQX), an antagonist of non-N-methyl-D-aspartate (nonNMDA) glutamate receptors, into the ipsilateral vestibular nucleus. All these findings suggest that the vestibular information activated the histaminergic neurons via the activation of the cholinergic neurons and this neuronal circuit was involved in the vestibulo-autonomic response.[28]

\section{CONCLUSION}

Vestibular stimulation modulates the neuro-transmitters which are involved in brain aging and delay aging. Hence we recommend controlled vestibular stimulation to all. This in the need of time to identify the importance of vestibular system and to start translational research in this area.

\section{REFERENCES}

1. Elie Hammam, Tay Dawood, Vaughan G, Macefield. Low frequency galvanic vestibular stimulation evokes two peaks of modulation in skin sympathetic nerve activity. June 2012, Volume 219, Issue 4, pp 441-446.

2. Lotta Winter et al. Vestibular stimulation on a motionsimulator impacts on mood states. Frontiers Psychology; November 2012; volume 3; article 499.

3. K.G. Akbulut, S Guney, F Cetin, H N Akgun, S H Actas, H Akbulut. Melatonin decreases brain aging by decreasing nitric oxide level. Neurophysiology. 2013; 45(3): 187-192.

4. Rubin RT, Mandell AJ, Crandall PH. Corticosteroid responses to limbic stimulation in man: Localization of stimulus sites. Science. 1996; 153: 767-768.

5. Dubocovich ML. Melatonin receptors: role on sleep and circadian rhythm regulation. Sleep Med. 2007 Dec; 8 Suppl 3: 34-42.

6. R Peters. Ageing and brain. Postgrad Med J. 2006 February; 82(964): 84-88.

7. Trollor J, Valenzuela M. Brain ageing in the new millennium. Austr N Z J Psychiatry 2001.35788-805.805.

8. Murphy D, DeCarli C, McIntosh A. et al Sex differences in human brain morphometry and metabolism: an in vivo quantitative magnetic resonance imaging and positron emission tomography study on the effect of ageing. Arch Gen Psychiatry 1996.53585-594.594.

9. Compton J, Van Amelsoort T, Murphy D. HRT and its effect on normal ageing of the brain and dementia. Br J Clin Pharmacol 2001.52647-653.653.

10. Parkin A. Memory and amnesia. Oxford: Blackwall, 1997.

11. Nyberg L, Bäckman L. Cognitive ageing: a view from brain 
imaging. In: Dixon R, Bäckman L, Nilsson L, eds. New frontiers in cognitive ageing. Oxford: Oxford University Press, 2004. 135-160.160.

12. Mukherjee J, Christian B, Dunigan K. et al Brain imageing of $18 \mathrm{~F}-$ Fallypride in normal volunteers: blood analysis, distribution, test-retest studies, and preliminary assessment of sensitivity to ageing effects on dopamine D-2/D-3 receptors. Synapse 2002.46170-188.188.

13. Compton J, Van Amelsoort T, Murphy D. HRT and its effect on normal ageing of the brain and dementia. $\mathrm{Br} \mathrm{J}$ Clin Pharmacol 2001.52647-653.653.

14. Goldberg S, Smith GS, Barnes A, Ma Y, Kramer E, Robeson K, Kirshner M, Pollock BG, Eidelberg D. Serotonin modulation of cerebral glucose metabolism in normal aging. Neurobiol Aging. 2004 Feb; 25(2):167-74.

15. McEntee WJ, Crook TH. Serotonin, memory and brain ageing. Psychopharmacology (Berl). 1991;103(2):143-9.

16. Mattson M, Maudsley S, Martin B. BDNF and 5-HT: a dynamic duo in age-related neuronal plasticity and neurodegenerative disorders. Trends Neurosci 2004. 27589-594.594.

17. Volchegorskii I, Shemyakov S, Turygin V. et al The age dynamics of monoamine oxidase activity and levels of lipid peroxidation products in the human brain. Neurosci Behav Physiol 2004.34303-305.305.

18. Toescu E, Verkhratsky A, Landfield P. Ca2+ regulation and gene expression in normal brain ageing. Trends Neurosci 2004. 27614-620.620.

19. Melov S. Modeling mitochondrial function in ageing neurons. Trends Neurosci 2004. 27601-606.606.

20. Carolyn Cidis Meltzer, Paul T. Francis. Brain aging and research at the close of 20th century: from bench to bedside. Dialogues Clin Neurosci. 2001 September; 3(3): 167-180.
21. Sailesh KS, Mukkadan JK. Vestibular modulation of endocrine secretions - A review. Int J Res Health Sci. 2014; In press.

22. Kumar Sai Sailesh, J.K.Mukkadan Can Controlled Vestibular Stimulation reduce stress -A Review." Health sciences. 2013; 2(3):js001.

2

http://www.ncbi.nlm.nih.gov/gene?Db=gene\&Cmd=ShowD etailView\&TermToSearch $=15$.

24. Sailesh KS, Mukkadan JK. Vestibular modulation of endocrine secretions -A review. Int J Res Health Sci. 2014; In press.

25. Ghazaleh Samoudi, Hans Nissbrandt, Mayank B. Dutia, Filip Bergquist. Noisy galvanic vestibular stimulation promotes GABA release in the substansia nigra and improves locomotion in hemi parkinsonin rats. Plos One.January 2012 | Volume 7 | Issue 1 | e29308.

26. A. V. Terry J R, J J Buccafusco.The cholinergic hypothesis of age and alzhimer's disease-related cognitive defects: recent challenges and their implications for novel drug development. JPET September 2003; vol. 306 no. 3: 821-827.

27. Buccafusco JJ and Terry AV Jr. Multiple CNS targets for eliciting beneficial effects on memory and cognition. J Pharmacol Exp The. 2000:295: 438-446.

28. Horii A, Takeda N, Mochizuki T, Okakura-Mochizuki K, Yamamoto Y. Effects of vestibular stimulation on acetylcholine release from rat hippocampus: an in vivo microdialysis study. J Neurophysiol. 1994 Aug; 72(2): 60511.

29. Horii A, Takeda N, Mochizuki T, Okakura-Mochizuki K, Yamamoto Y, Yamatodani A, Kubo T. Vestibular modulation of the septo-hippocampal cholinergic system of rats. Acta Otolaryngol Suppl. 1995; 520 Pt 2: 395-8. 